\title{
A Comparison of Human Bone Marrow-Derived Mesenchymal Stem Cells and Human Umbilical Cord-Derived Mesenchymal Stromal Cells for Cartilage Tissue Engineering
}

\author{
Limin Wang, Ph.D., ${ }^{1, *}$ Ivy Tran, ${ }^{2,{ }^{*}}$ Kiran Seshareddy, B.V.Sc., A.H., ${ }^{3}$ \\ Mark L. Weiss, Ph.D., ${ }^{3}$ and Michael S. Detamore, Ph.D. ${ }^{4}$
}

Bone marrow-derived mesenchymal stem cells (BMSCs) have long been considered the criterion standard for stem cell sources in musculoskeletal tissue engineering. The true test of a stem cell source is a side-by-side comparison with BMSCs. Human umbilical cord-derived mesenchymal stromal cells (hUCMSCs), one such candidate with high potential, are a fetus-derived stem cell source collected from discarded tissue (Wharton's jelly) after birth. Compared with human BMSCs (hBMSCs), hUCMSCs have the advantages of abundant supply, painless collection, no donor site morbidity, and faster and longer self-renewal in vitro. In this 6-week study, a chondrogenic comparison was conducted of hBMSCs and hUCMSCs in a three-dimensional (3D) scaffold for the first time. Cells were seeded on polyglycolic acid (PGA) scaffolds at $25 \mathrm{M}$ cells $/ \mathrm{mL}$ and then cultured in identical conditions. Cell proliferation, biosynthesis, and chondrogenic differentiation were assessed at weeks 0, 3, and 6 after seeding. At weeks 3 and 6, hUCMSCs produced more glycosaminoglycans than hBMSCs. At week 6, the hUCMSC group had three times as much collagen as the hBMSC group. Immunohistochemistry revealed the presence of collagen types I and II and aggrecan in both groups, but type II collagen staining was more intense for hBMSCs than hUCMSCs. At week 6, the quantitative reverse transcriptase polymerase chain reaction (RTPCR) revealed less type I collagen messenger RNA (mRNA) with both cell types, and more type II collagen mRNA with hBMSCs, than at week 3 . Therefore, it was concluded that hUCMSCs may be a desirable option for use as a mesenchymal cell source for fibrocartilage tissue engineering, based on abundant type I collagen and aggrecan production of hUCMSCs in a 3D matrix, although further investigation of signals that best promote type II collagen production of hUCMSCs is warranted for hyaline cartilage engineering.

\section{Introduction}

$\mathbf{M}$ ESENCHYMAL STEM CELLS (MSCs) can differentiate along a variety of cell lineages that can be used for tissue engineering and regenerative medicine. MSCs reside in many tissues, including bone marrow, ${ }^{1}$ adipose tissue, ${ }^{2}$ blood, ${ }^{3}$ synovial fluid, ${ }^{4}$ dermis, ${ }^{5}$ muscle, ${ }^{6}$ and dental pulp. ${ }^{7}$ Human bone marrow-derived MSCs (hBMSCs) are the most commonly used MSCs for scientific and clinical purposes. They are isolated via plastic adhesion or negative selection from bone marrow aspirate that includes a highly heterogeneous cell population such as hematopoietic cells, endothelial cells, and adipocytes. ${ }^{8}$ However, there are some limitations of hBMSCs. The relative number of hBMSCs in the marrow and their differentiation potential decrease significantly with age. ${ }^{9}$ Moreover, the harvesting procedure is painful and invasive and may lead to complications and morbidity. ${ }^{10}$ Because of the disadvantages associated with hBMSCs, efforts have been made to find alternative sources of MSCs that function as well as hBMSCs but overcome these key limitations.

In recent years, umbilical cord-derived mesenchymal stromal cells (UCMSCs) have been explored as a MSC source with clear advantages over hBMSCs. UCMSCs are extracted through enzyme digestion or the explant culture method from Wharton's jelly of umbilical cords, ${ }^{11}$ a discarded tissue

\footnotetext{
${ }^{1}$ Departments of Biomedical Engineering and ${ }^{2}$ Chemistry, University of Michigan, Ann Arbor, Michigan.

${ }^{3}$ Department of Anatomy and Physiology, Kansas State University, Manhattan, Kansas.

${ }^{4}$ Department of Chemical and Petroleum Engineering, University of Kansas, Lawrence, Kansas.

*Limin Wang and Ivy Tran have contributed equally to this work and were considered first co-authors.
} 
in abundant supply. The harvesting procedure is not invasive or painful, and there is no donor site morbidity. The enzyme extraction yields $10 \times 10^{3}$ to $50 \times 10^{3}$ cells per cm of cord, ${ }^{12,13}$ corresponding to approximately $2 \times 10^{5}$ to $10 \times 10^{5}$ primary cells per cord, and these primary cells can be expanded 300 times as much as the original number for more than seven passages without the loss of differentiation potential. ${ }^{13}$ hUCMSCs strongly express surface markers that have been identified with other MSCs, including CD10, ${ }^{12}$ CD13, ${ }^{12,14-16}$ CD29, ${ }^{12,14,15,17}$ CD $44,{ }^{12-18}$ CD $49 \mathrm{e}^{12,19}$ CD51, ${ }^{17}$ CD73/ SH3 ${ }^{13,14,16-18}$ CD90 (Thy-1) ${ }^{12,15,16,18,19}$ CD105/SH2, ${ }^{12-14,16-18}$ CD106, ${ }^{14} \mathrm{CD} 117,{ }^{16,18,20} \mathrm{CD} 146,{ }^{19} \mathrm{CD} 166,{ }^{14-16}$ and human leukocyte antigen (HLA)-1/HLA-ABC, ${ }^{12,14,15,18}$ and are negative for hematopoietic markers (e.g., CD14, CD31, CD34, CD38, CD45, HLA-DR). ${ }^{12-25}$ Moreover, the presence of Oct-4, Nanog, and Sox-2 transcription factors indicates that a subpopulation of UCMSCs might share some properties of embryonic and non-embryonic stem cells. ${ }^{23}$ hUCMSCs have the ability to differentiate into mesenchymal cell lineages, such as osteogenic, chondrogenic, myogenic, and adipogenic. ${ }^{13,17-19,25}$ Moreover, the number of fibroblast colony-forming units is significantly higher in hUCMSCs than hBMSCs. ${ }^{14}$

In the past 4 years, only five studies have demonstrated the chondrogenic differentiation of hUCMSCs in cell pellet culture $^{13,17,19}$ or poly(glycolic acid) (PGA) scaffolds. ${ }^{26,27}$ Wang et al. ${ }^{17}$ first revealed evidence of chondrogenesis by a positive immunohistochemical staining of type II collagen, which was also observed with non-differentiated cells. Other than a conference abstract, ${ }^{28}$ our group was the first to apply hUCMSCs in three-dimensional (3D) musculoskeletal tissue engineering. ${ }^{26,27}$ In these studies, we demonstrated that $3 \mathrm{D}$ culture of hUCMSCs in PGA scaffolds led to a fibrocartilage with intense type I collagen and moderate collagen type II and aggrecan immunostaining. ${ }^{26,27}$ Here, our objective is to compare hUCMSCs and hBMSCs in vitro in a cartilage tissue engineering application. Both types of cells were cultured in PGA scaffolds, and their proliferation, biosynthesis, and chondrogenic differentiation were evaluated over a period of 6 weeks.

\section{Materials and Methods}

\section{Cell harvest}

hUCMSCs were harvested following our previous methods, with institutional review board (IRB) approval and informed consent. ${ }^{12}$ The cells were plated and recorded as passage 0 (P0) in 6-well plates containing a low-serum medium at a density of 10,000 cells $/ \mathrm{cm}^{2}$. The medium consisted of low-glucose Dulbecco's modified Eagle medium (DMEMLG; Invitrogen, Carlsbad, CA) and MCDB-201 medium (Sigma, St. Louis, MO) supplemented with $1 \times$ insulintransferrin-selenium (ITS; Invitrogen), 0.15\% lipid-rich bovine serum albumin (Albumax; Invitrogen), $0.1 \mathrm{nM}$ dexamethasone (Sigma), 10 $\mu \mathrm{M}$ ascorbic acid-2-phosphate (Sigma), $1 \times$ penicillin/streptomycin (Fisher Scientific, Pittsburgh, PA), $2 \%$ fetal bovine serum (FBS; Invitrogen), $10 \mathrm{ng} / \mathrm{mL}$ recombinant human epidermal growth factor (Invitrogen), and $10 \mathrm{ng} / \mathrm{mL}$ human platelet-derived growth factor BB (R\&D Systems, Minneapolis, MN). Cells in the well plates were fed every 2 to 3 days and maintained in a cell culture incubator (NuAire, Autoflow, 5\% carbon dioxide, $37^{\circ} \mathrm{C}, 90 \%$ humidity). Cells were then detached at $80 \%$ to $90 \%$ confluence and plated into $25-\mathrm{cm}^{2}$ flasks. At P1, cells were resuspended at the density of 1 million cells per $\mathrm{mL}$ of freezing medium composed of $90 \%$ FBS and $10 \%$ dimethyl sulfoxide (DMSO; Fisher Scientific). The cell suspension was transferred into cryotubes, which were stored in Mr. Frosty freezing containers (Nalgene, Rochester, NY) at $-80^{\circ} \mathrm{C}$ overnight and then transferred to a liquid nitrogen cryogenic storage system at $-196^{\circ} \mathrm{C}$ for future use. P0 frozen hBMSCs were obtained from StemCell Technologies (Vancouver, Canada), with IRB approval. The bone marrow was extracted from the posterior iliac crest from a maximum of four sites per donor ( $25 \mathrm{~mL} / \mathrm{site})$. The phenotype of P0 hBMSC was evaluated using flow cytometry, and cells included CD29 (>90\%), CD44 (>90\%), CD105 (>90\%), CD166 (>90\%), CD14 (<1\%), CD34 $(<1 \%)$, and CD45 $(<1 \%)$.

\section{Cell seeding}

Both types of cells were thawed and expanded with a plating density of 5000 to 6000 cells $/ \mathrm{cm}^{2}$ in $300-\mathrm{cm}^{2}$ flasks to P4 in a medium containing DMEM-LG, 10\% FBS (StemCell Technologies), 1\% penicillin/streptomycin (Invitrogen), and $1 \%$ non-essential amino acids (Invitrogen). Non-woven PGA meshes $(50 \mathrm{mg} / \mathrm{cc}$; Synthecon, Houston, TX) were punched to cylindrical scaffolds with a 5-mm diameter and 2-mm thickness and then sterilized with ethylene oxide. After sterilization, the scaffolds were aired under a fume hood for 1 day, then wetted with sterile filtered ethanol and two washes of sterile PBS. The scaffolds were then soaked in the medium for 1 day, and the medium was removed for cell seeding. Both cell types, at P4, were seeded at 20 million cells per $\mathrm{mL}$ of scaffold via orbital shakers onto PGA scaffolds at $150 \mathrm{rpm}$ for $24 \mathrm{~h}$. Finally, the medium was replaced with $2 \mathrm{~mL}$ of chondrogenic differentiation medium consisting of high-glucose DMEM (DMEM-HG; Invitrogen), 1\% nonessential amino acids, $1 \times$ ITS premix (BD Biosciences, San Jose, CA), $10 \mathrm{ng} / \mathrm{mL}$ transforming growth factor beta-1 (TGF$\beta 1$; PeproTech, Rocky Hill, NJ), $100 \mathrm{nM}$ dexamethasone (Sig$\mathrm{ma}), 50 \mu \mathrm{g} / \mathrm{mL}$ ascorbic acid 2-phosphate (Sigma), $100 \mathrm{mM}$ sodium pyruvate (Fishersci), and $40 \mu \mathrm{g} / \mathrm{mL}$ L-proline (Sigma). This time point was recorded as week 0 . Medium was changed every other day over a period of 6 weeks.

\section{Biochemical analysis}

At weeks 0,3 , and 6 , constructs $(\mathrm{n}=4)$ were digested by adding $1.1 \mathrm{~mL}$ papain solution $(120 \mu \mathrm{g} / \mathrm{mL})$ at $60^{\circ} \mathrm{C}$ overnight and centrifuged at $10,000 \times g$ for $5 \mathrm{~min}$ to remove the PGA debris. The supernatant was stored at $-20^{\circ} \mathrm{C}$ for future assays. DNA content was measured using a PicoGreen kit (Invitrogen). A conversion factor of $8.5 \mathrm{pg}$ DNA/cell determined in preliminary studies can be used to convert DNA content to cell number. Biosynthesis was evaluated by measuring total glycosaminoglycan (GAG) and hydroxyproline (HYP) contents. GAG content was measured using a dimethylmethylene blue (DMMB) dye binding assay kit (Biocolor, Belfast, UK). From each sample, $100 \mu \mathrm{L}$ was added to $1 \mathrm{~mL}$ of DMMB and allowed to bind for $30 \mathrm{~min}$. Solutions were then centrifuged, supernatant was discarded, and the pellet was resuspended and read at $656 \mathrm{~nm}$. HYP content was determined using a modified HYP assay. ${ }^{29}$ Briefly, $400 \mu \mathrm{L}$ of each sample was hydrolyzed with an equal volume of $4 \mathrm{~N}$ sodium hydroxide at $121^{\circ} \mathrm{C}$ for $30 \mathrm{~min}$, neutralized with an equal volume of $4 \mathrm{~N}$ hydrochloric acid, and then titrated to an approximate $\mathrm{pH}$ 
range between 6.5 and 7.0. One $\mathrm{mL}$ of this solution was combined with $0.5 \mathrm{~mL}$ chloramine- $\mathrm{T}(14.1 \mathrm{~g} / \mathrm{L})$ in the buffer $(50 \mathrm{~g} / \mathrm{L}$ citric acid, $120 \mathrm{~g} / \mathrm{L}$ sodium acetate trihydrate, $34 \mathrm{~g} / \mathrm{L}$ sodium hydroxide, and $12.5 \mathrm{~g} / \mathrm{L}$ acetic acid), and the resulting solution was then combined with $0.5 \mathrm{~mL}$ of $1.17 \mathrm{mM}$ p-dimethylaminobenzaldehyde in perchloric acid and read at $550 \mathrm{~nm}$. A conversion factor of 11.5 can be used to convert HYP mass to collagen mass based on our preliminary studies.

\section{Hematoxylin staining and immunohistochemistry for types I and II collagen and aggrecan}

Scaffolds were frozen and sliced into thin sections $(10 \mu \mathrm{m})$ that were fixed in chilled acetone $\left(4^{\circ} \mathrm{C}\right)$ for $10 \mathrm{~min}$ before staining. Hematoxylin staining was performed using VECTOR hematoxylin QS (Vector Laboratories, Burlingame, CA), according to the manufacturer instructions. In brief, slides were rinsed in tap water and immersed in the hematoxylin reagent for $2 \mathrm{~min}$. After the staining, slides were rinsed in tap water, dehydrated, and mounted. Immunohistochemical analysis $(n=2)$ was performed in a BioGenex i6000 autostainer (BioGenex, San Ramon, CA). Slides were rehydrated with PBS for $5 \mathrm{~min}$. Endogenous peroxidase activity was inhibited using 1\% hydrogen peroxide in methanol for $30 \mathrm{~min}$. Each section was blocked in 3\% horse serum for $20 \mathrm{~min}$ and incubated with a primary antibody for 1 hour. Primary antibodies used in this study included the mouse monoclonal immunoglobulin (Ig)G anti-collagen I (1:1500 dilution; Accurate Chemical and Scientific, Westbury, NY), mouse monoclonal IgG anti-collagen II (1:1000 dilution; Chondrex, Redmond, WA), and mouse monoclonal IgG anti-aggrecan (1:50 dilution; Abcam, Cambridge, MA). After primary antibody incubation, the sections were incubated with a streptavidin-linked horse anti-mouse IgG secondary antibody (Vector Laboratories) for $30 \mathrm{~min}$. After secondary antibody incubation, the sections were incubated with an avidin-biotinylated enzyme complex (ABC complex; Vector Laboratories) for $30 \mathrm{~min}$, and then VIP substrate (purple color) (Vector Laboratories) was applied on sections for $6 \mathrm{~min}$. Protocols run with the primary antibody omitted served as negative controls.

\section{RNA isolation and gene expression analysis}

Real-time reverse transcriptase polymerase chain reaction (RT-PCR) was used to quantify the relative gene expression level of types I and II collagen and aggrecan. The constructs $(n=4)$ were collected at weeks 3 and 6 and homogenized with an electrical homogenizer in $1 \mathrm{~mL}$ of Trizol reagent (Invitrogen) to extract messenger RNA (mRNA) according to the manufacturer's protocol. Total mRNA was reverse transcribed to complementary DNA (cDNA) using a highcapacity cDNA Archive kit (Applied Biosystems, Foster City, CA). Real-time RT-PCR reactions were run in an Applied Biosystems 7500 Fast Sequence Detection System. TaqMan gene expression assay kits, including two pre-designed primers and one probe, were used for transcript levels of the proposed genes. Two $\mu \mathrm{L}$ of cDNA from each sample was mixed with $7 \mu \mathrm{L}$ of RNase/DNase free water, $1 \mu \mathrm{L}$ of $20 \times$ TaqMan gene expression assay reagent, and $10 \mu \mathrm{L}$ of $2 \times$ TaqMan universal PCR master mix. Assay IDs of TaqMan gene expression assays were Hs00164004_m1 for type I collagen, Hs00156568_m1 for type II collagen, Hs00153936_m1 for aggrecan, and Hs99999905_m1 for glyceraldehyde 3phosphate dehydrogenase (GAPDH). Relative mRNA expression level for each target gene was evaluated using a $2^{-\Delta \Delta \mathrm{Ct}}$ method. ${ }^{30}$ The $\mathrm{Ct}$ values of target genes were first normalized by subtracting the Ct values of the TaqMan human housekeeping gene GAPDH to obtain $\Delta \mathrm{Ct}$ values. They were then normalized by subtracting the $\mathrm{Ct}$ value of the calibrator sample, the hBMSC group at week 3, to obtain $\Delta \Delta \mathrm{Ct}$ values, which were finally calculated to acquire the fold changes. Each sample was analyzed in triplicate.

\section{Statistical analysis}

All data were expressed as means \pm one standard deviation and analyzed using analysis of variance (ANOVA) followed by Tukey's honestly significant difference (HSD) post hoc tests. Two-way ANOVAs with interaction were performed with time-points and cell sources as statistical factors. A statistical threshold of $p<0.05$ was used to indicate whether there were statistically significant differences between different groups.

\section{Results}

\section{Seeding efficiency and DNA content}

At week 0, a seeding efficiency (defined as the percentage ratio of the DNA content of attached cells to the DNA

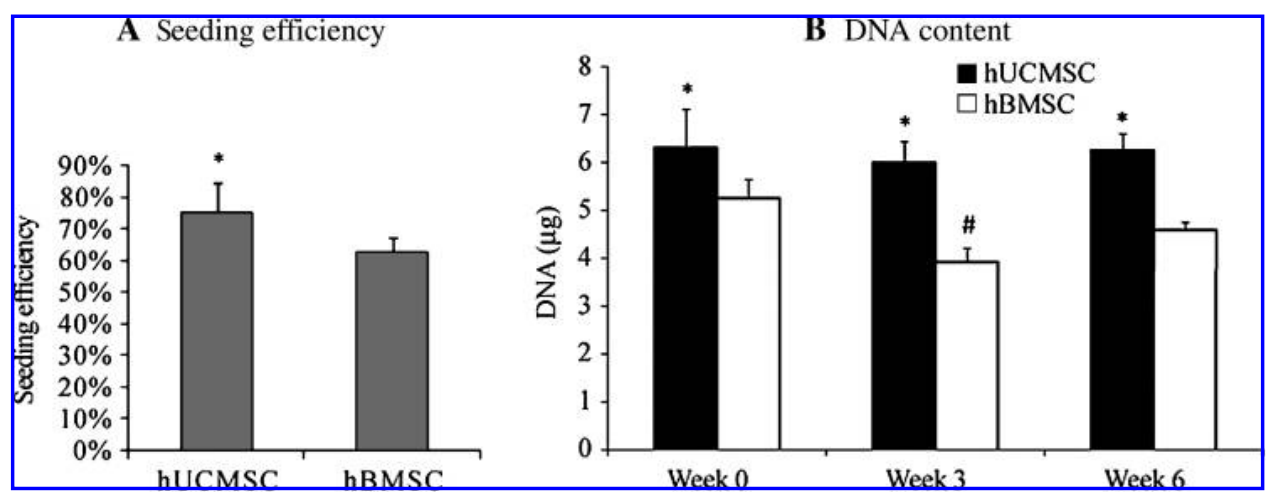
converted to cell number using a conversion factor of $8.5 \mathrm{pg}$ DNA/cell. Error bars represent standard deviations.

FIG. 1. Seeding efficiency at week $0(\mathbf{A})$ and DNA content at weeks 0,3 , and 6 (B) $(\mathrm{n}=4)$. Human umbilical cord-derived mesenchymal stromal cells (hUCMSCs) outperformed human bone marrow-derived mesenchymal stem cells (hBMSCs) in seeding efficiency and in DNA content at all time points. *Statistically significant difference 
FIG. 2. Glycosaminoglycan (GAG) and hydroxyproline (HYP) content per construct and per unit DNA at weeks 0,3 , and $6(n=4)$. Human umbilical cord-derived mesenchymal stromal cells (hUCMSCs) outperformed human bone marrow-derived mesenchymal stem cells (hBMSCs) in GAG and HYP biosynthesis, most notably with nearly three times as much collagen per construct at week 6 as evidenced by HYP content. *Statistically significant difference between hUCMSC and hBMSC groups at a specific time point. \#Statistically significant difference from week $0 \mathrm{hUCMSC}$ or hBMSC group. @Statistically significant difference between weeks 3 and 6 in hUCMSC or hBMSC group. Error bars represent standard deviations.

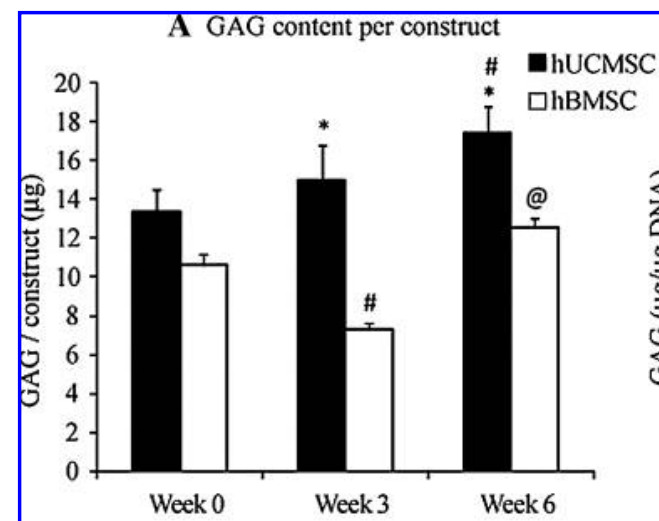

C HYP content per construct

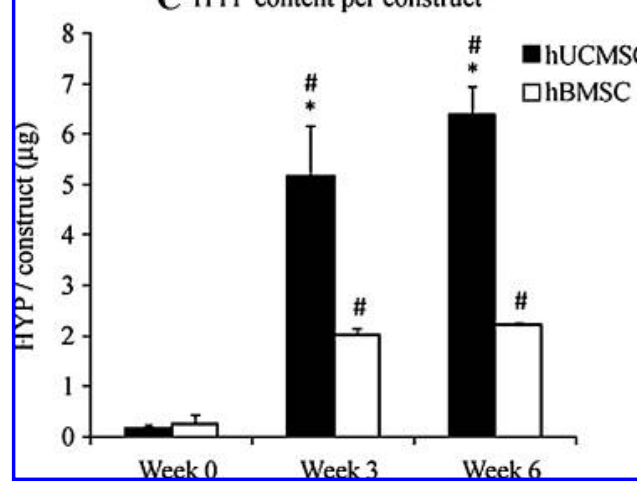

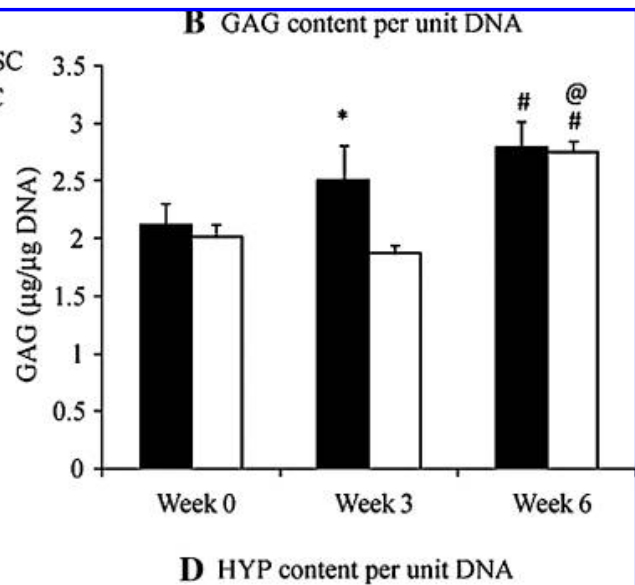

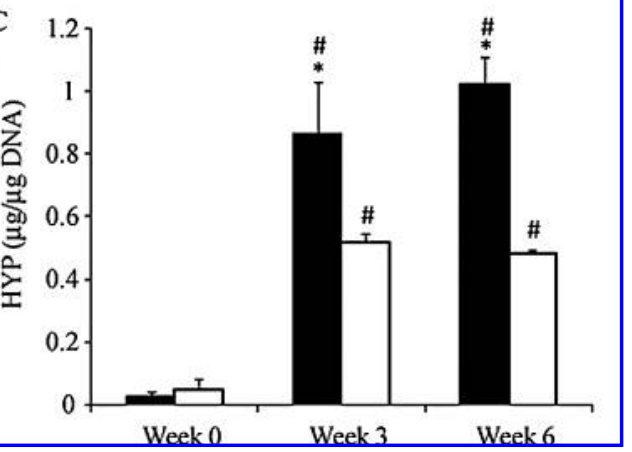

content of seeded cells) of $75 \%$ was achieved with hUCMSCs, whereas a seeding efficiency of only $62 \%$ was observed with hBMSCs $(p<0.05)$ (Fig. 1A). After the initial seeding period, the hUCMSC and hBMSC groups both maintained their DNA content over 6 weeks, except for a drop $(p<0.05)$ at week 3 in the hBMSC group. However, after the drop, a recovery increase in DNA content occurred without significant difference. In comparison, the DNA content in the hUCMSC group was significantly higher than in the hBMSC group throughout the culture period $(p<0.05)$ (Fig. 1B).

\section{GAG and HYP content}

There was a continuing increase in GAG content per construct in the hUCMSC group over 6 weeks $(p<0.05)$, whereas the GAG content decreased in the hBMSC group from weeks 0 to $3(p<0.05)$ and then increased from weeks 3 to $6(p<0.05)$ (Fig. 2A). However, the increases did not lead to a significant difference between weeks 0 and 6 for either group. The hUCMSC group had a $51 \%$ and $28 \%$ higher GAG content per construct than the hBMSC group at weeks 3 and 6, respectively $(p<0.05)$. With regard to GAG content per unit DNA, the hUCMSC and hBMSC groups both had a significant increase between weeks 0 and 6 (Fig. 2B) $(p<0.05)$. There was also a significant increase in GAG content for the hBMSC group from weeks 3 to $6(p<0.05)$. The hUCMSC group at week 3 had a higher GAG content per unit DNA than the hBMSC group $(p<0.05)$, although there was no statistically significant difference between them at week 6 .

HYP content per construct and per unit DNA increased markedly from weeks 0 to 3 in the hUCMSC and hBMSC groups (Fig. 2C, D), whereas there was no increase in HYP

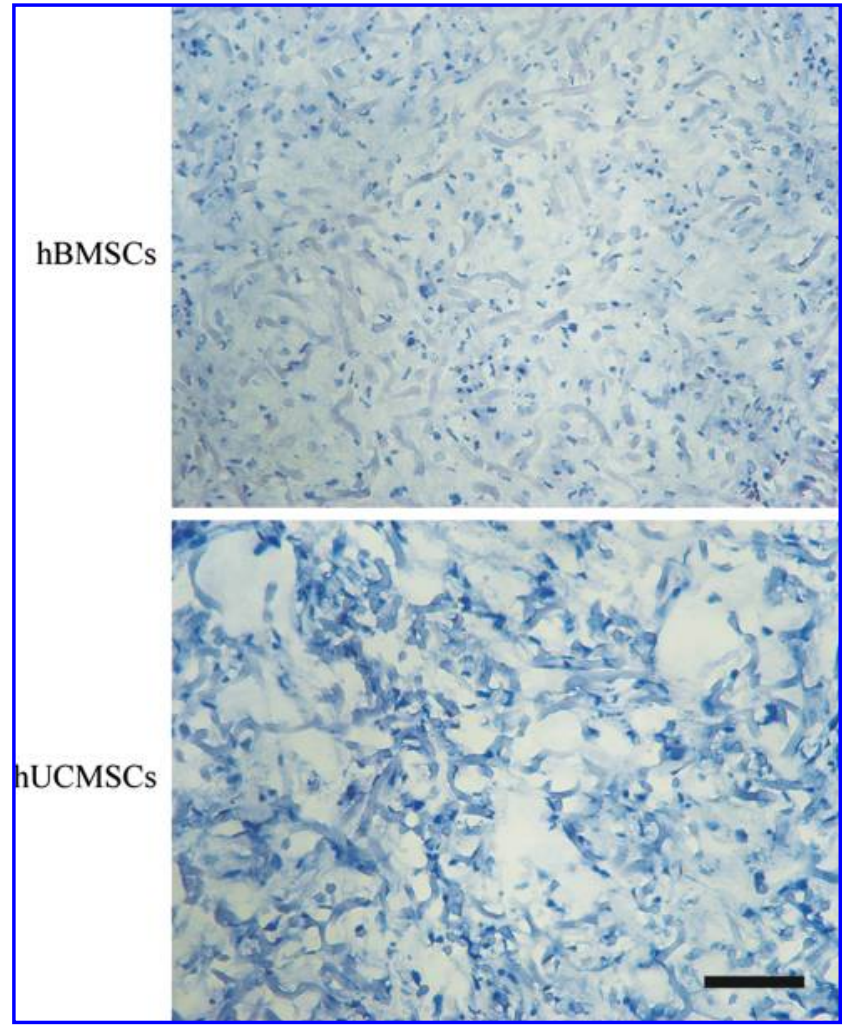

FIG. 3. Hematoxylin staining $(n=2)$. The scale bar is $100 \mu \mathrm{m}$. The human bone marrow-derived mesenchymal stem cell (hBMSC) group had a higher cell density than the human umbilical cord-derived mesenchymal stromal cell (hUCMSC) group. Color images available online at www .liebertonline.com/ten. 
content between week 3 and 6 for either group. The hUCMSC group had 2.5 and 2.9 times as much HYP per construct as the hBMSC group at weeks 3 and 6, respectively $(p<0.05)$. Similar to HYP content per construct, the HYP content per unit DNA increased from weeks 0 to $3(p<0.05)$ and then had no significant changes until week 6 for both groups. The hUCMSC group possessed 1.7 and 2.1 times as much HYP per unit DNA as the hBMSC group $(p<0.05)$.

\section{Histology and immunohistochemistry}

At week 6, hematoxylin staining revealed that the hBMSC group had a higher cell density (cell number per volume) than the hUCMSC group (Fig. 3). The PGA debris density in the hBMSC group was higher than that of the hUCMSC group as well. Immunohistochemistry revealed positive staining for types I and II collagen and aggrecan in the hUCMSC and hBMSC groups through the 6-week period (Fig. 4). At week 3, the hBMSC and hUCMSC groups both showed an abundant amount of type I collagen, a small amount of type II collagen, and a moderate amount of aggrecan. Stronger staining of type I collagen and aggrecan was present in the hUCMSC group than in the hBMSC group. At week 6, the hUCMSC and hBMSC groups presented a concentrated staining for type I collagen and a more-intense staining for aggrecan than at week 3 . There was a vast increase in type II collagen staining in the hBMSC group from weeks 3 to 6 , whereas only a trace of type II collagen was detected in the hUCMSC group at week 6 .

\section{RT-PCR analysis}

hUCMSCs had a lower mRNA level of type I collagen than hBMSCs at weeks 3 and $6(p<0.05)$, a lower mRNA level of type II collagen at week 6 , and the same level of aggrecan at weeks 3 and 6 (Fig. 5). From weeks 3 and 6, in the hUCMSC and hBMSC groups, type I collagen mRNA level decreased $(p<0.05)$, and aggrecan mRNA level remained at the same level. Type II collagen mRNA level increased 3.8 times in the hBMSC group from weeks 3 to 6 $(p<0.05)$, whereas no change was observed in the hUCMSC group.

\section{Discussion}

It has been previously shown that hUCMSCs can differentiate toward a chondrogenic lineage. ${ }^{13,17,19,26}$ However, the application of these cells in musculoskeletal tissue engineering has scarcely been investigated. ${ }^{26-28}$ This study, for the first time, specifically focused on the comparison of hUCMSCs and hBMSCs in a biomaterial-based 3D environment. This comparison is vital to the validation of hUCMSCs as a formidable cell source for cartilage-and more broadly, musculoskeletal-tissue engineering.

It has been reported that hUCMSCs have a faster proliferation rate in monolayer culture than adult stem cells. ${ }^{31}$ Indeed, in the current study, hUCMSCs reached $80 \%$ to $90 \%$ confluence 2 to 3 days earlier than hBMSCs, and both cell types had similar cell numbers per flask at this confluence.

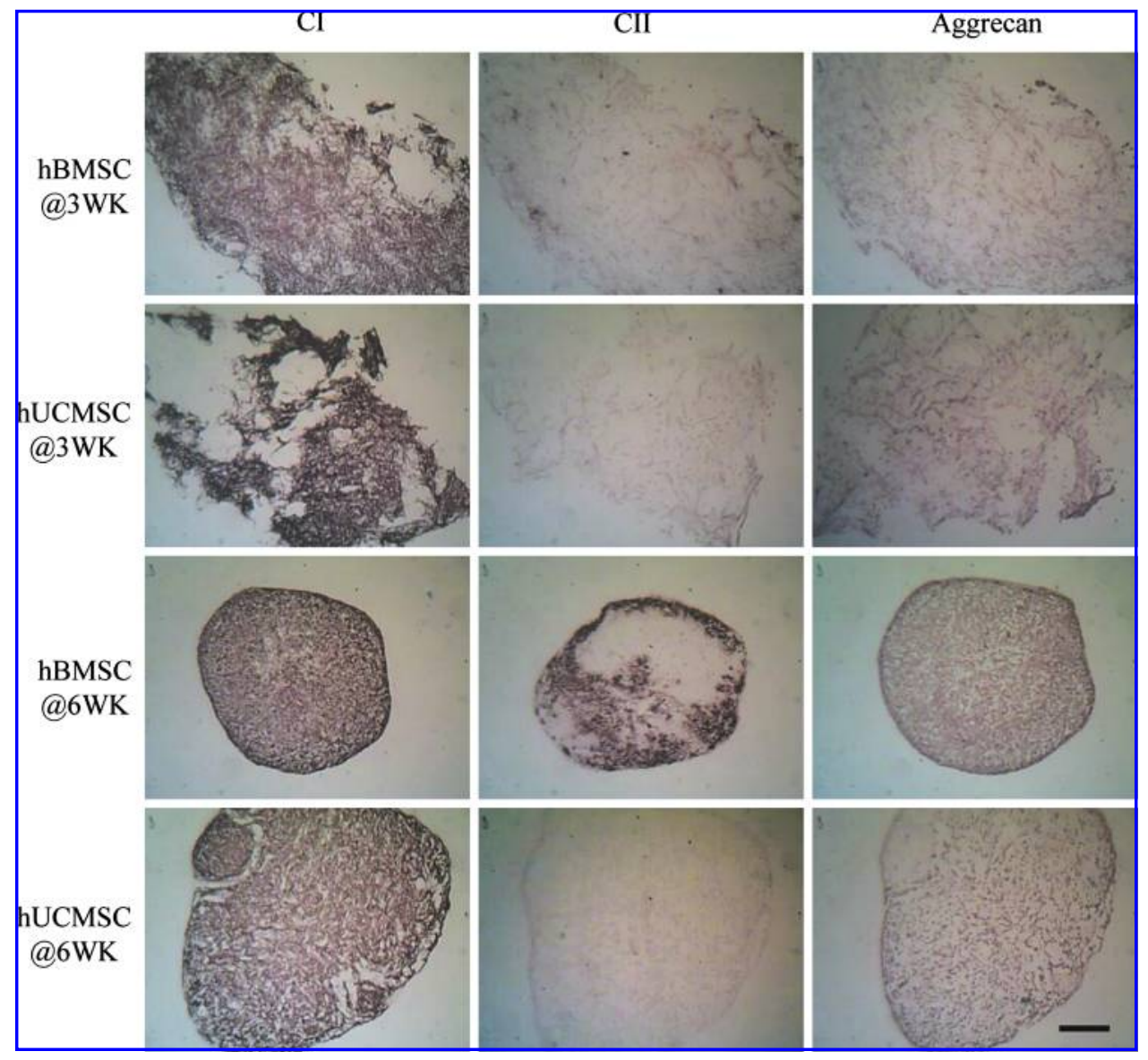

FIG. 4. Immunohistochemical staining for types I and II collagen, and aggrecan $(\mathrm{n}=2)$. Although collagen I staining was comparable between human umbilical cord-derived mesenchymal stromal cells (hUCMSCs) and human bone marrowderived mesenchymal stem cells (hBMSCs), collagen II staining was more intense with hBMSCs. The scale bar is $500 \mu \mathrm{m}$. CI, type I collagen; CII, type II collagen; @3WK, at week 3; @6WK, at week 6. Negative control groups (not shown) confirmed the absence of non-specific staining. Color images available online at www.liebertonline .com/ten. 


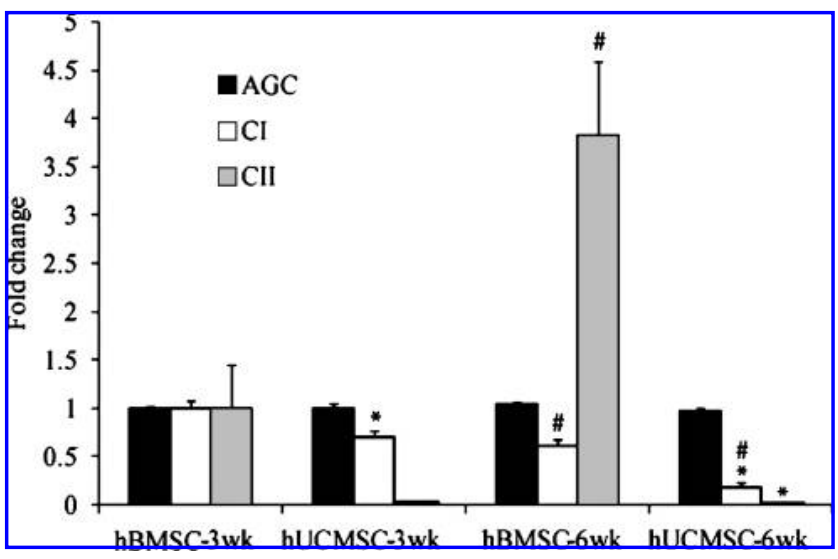

FIG. 5. Gene expression for types I and II collagen, and aggrecan $(n=4)$. Human bone marrow-derived mesenchymal stem cells (hBMSCs) significantly outperformed human umbilical cord-derived mesenchymal stromal cells (hUCMSCs) in collagen II gene expression at the week 6 snapshot. CI, type I collagen; CII, type II collagen. *Statistically significant difference between hUCMSC and hBMSC groups at a specific time point. \#Statistically significant difference between weeks 3 and 6 in hUCMSC or hBMSC group.

The faster proliferation provides for a larger number of cells in a shorter time period to better meet the needs of tissue engineering and clinical practice. The higher seeding efficiency with the hUCMSCs at week 0 indicated that hUCMSCs were more adherent to PGA scaffolds than hBMSCs. This higher seeding efficiency resulted in a higher DNA content (cell number) for the hUCMSC group, which was maintained over 6 weeks and probably contributed in part to the higher matrix production in the hUCMSC group. Although the cell density in the hBMSC group was higher than in the hUCMSC group, as revealed by hematoxylin staining (Fig. 3), smaller scaffold volume with the hBMSC constructs than with the hUCMSC constructs, as revealed by the immunohistochemical staining, explains this seemingly conflicting result (Fig. 4). The decrease in DNA content with hBMSCs from weeks 0 to 3 may be due to the degradation of the PGA scaffold, as we have noted in the past. $^{27,32}$ The hUCMSC group also experienced a slight loss of DNA (not statistically significant). The ability of hUCMSCs to proliferate at a higher rate and the higher matrix content that helps to retain cells in scaffolds can explain the maintenance of DNA content with hUCMSCs. To overcome cell loss caused by PGA degradation, slowly degrading scaffolds such poly-L-lactic acid and poly-lactic-co-glycolic acid will be investigated in the future.

In this study, the hUCMSC group produced more GAGs than the hBMSC group. The higher GAG content per construct with hUCMSCs was due to the larger number of cells, because the GAG content per unit DNA at week 6 was similar for the two groups. With regard to collagen production, the hBMSC and hUCMSC groups both showed an increase in collagen content throughout the 6-week culture, with the largest increase occurring between weeks 0 and 3 . The higher porosity of cell-scaffold constructs in the first 3 weeks might contribute to higher collagen production, because it provides a large space for matrix synthesis and nutrient diffusion. When compared directly with each other, the hUCMSC group produced 2.5 and 3.5 times as much collagen per construct as the hBMSC group at weeks 3 and 6 , respectively. More importantly, the hUCMSC group had more collagen per unit DNA than the hBMSC group, indicating that hUCMSCs are better able to produce collagen than hBMSCs under the prescribed conditions. Thus, the high collagen content produced by hUCMSCs is attractive for cartilage tissue engineering.

Exposed to chondrogenic media, both types of cells produced a cartilage-like tissue with types I and II collagen and aggrecan. A down-regulation of type I collagen gene expression during this period accompanied the chondrogenic differentiation of both cell types. Higher type II collagen expression in the hBMSC group at the mRNA and protein levels suggested that chondrogenic differentiation was more extensive with hBMSCs under the prescribed conditions. Although the hBMSC group had a higher gene expression of types I and II collagen at week 6, the total collagen content in the hUCMSC group was higher than in the hBMSC group. Although at first glance these results may appear contradictory, the gene expression level represents a snapshot of the cellular disposition at that exact point in time, whereas the hydroxyproline assay is cumulative, representing the total collagen protein synthesized. The hydroxyproline data appear to be relatively consistent with the intensity and construct size with collagen immunostaining, which is also a cumulative assay. With regard to aggrecan, mRNA and protein expression were comparable between the two groups, which suggests that hUCMSCs were at least in one capacity matching the apparent chondrogenesis of hBMSCs.

In previous hUCMSC pellet culture, ${ }^{13,17}$ plentiful type II collagen $^{13,17}$ was observed with a small amount of type I collagen $^{13}$ around the periphery. Limited oxygen (hypoxia) exists in the center of cell pellets because of a low diffusion coefficient, ${ }^{33}$ which may have contributed to the production of type II collagen. ${ }^{34}$ It was unexpected that only a trace amount of type II collagen ${ }^{13}$ was observed in hBMSC pellets because hBMSCs generally produced dominant type II collagen in pellets. ${ }^{35}$ The constructs based on highly porous PGA scaffolds ( $>95 \%$ porosity) in the current study had better diffusion than cell pellets, especially at the early stages. Moreover, the differences in chondrogenic media (TGF- $\beta 1$ vs $\beta 3$ ) and harvesting methods might explain these conflicting results between the pellet culture in the literature and the biomaterial-based culture in this study.

In conclusion, hUCMSCs were seeded more efficiently and demonstrated superior biosynthesis, with nearly three times as much collagen production as hBMSCs. The differentiation profile of hUCMSCs, consisting of a large amount of type I collagen, a small amount of type II collagen, and moderate aggrecan content, clearly indicated that hUCMSCs may be highly desirable for fibrocartilage tissue engineering such as for the temporomandibular joint disc or the knee meniscus. In contrast, hBMSCs appeared to have progressed further down a chondrogenic lineage based on superior collagen II gene expression and protein production. However, one may expect that the hUCMSCs will require a modified set of signals to provide for optimal chondrogenesis. With the identification of this set of signals, it would be expected that hUCMSCs would be capable of producing a similar 
tissue in a drastically reduced time, which would translate to better patient care. From the perspective of clinical practice, hUCMSCs provide clear advantages over hBMSCs in that hUCMSCs are easily obtained from a discarded tissue, are in abundant supply, have no donor site morbidity, and are highly expendable before senescence or differentiation. Moreover, in the future, if parents cryogenically save part of their child's umbilical cord, these hUCMSCs would also be available as an autologous cell source later in life with all of the aforementioned advantages.

Based on these known advantages, and the results of this study, we therefore conclude that hUCMSCs are a highly desirable cell source for fibrocartilage tissue engineering and have the potential to surpass the criterion standard of hBMSCs in hyaline cartilage regeneration with further investigation of chondrogenic signals tailored to hUCMSCs.

\section{Disclosure Statement}

No competing financial interests exist.

\section{References}

1. Prockop, D.J. Marrow stromal cells as stem cells for nonhematopoietic tissues. Science 276, 71, 1997.

2. Zuk, P.A., Zhu, M., Ashjian, P., De Ugarte, D.A., Huang, J.I., Mizuno, H., Alfonso, Z.C., Fraser, J.K., Benhaim, P. and Hedrick, M.H. Human adipose tissue is a source of multipotent stem cells. Mol Biol Cell 13, 4279, 2002.

3. Childs, R., Chernoff, A., Contentin, N., Bahceci, E., Schrump, D., Leitman, S., Read, E.J., Tisdale, J., Dunbar, C., Linehan, W.M., Young, N.S. and Barrett, A.J. Regression of metastatic renal-cell carcinoma after nonmyeloablative allogeneic peripheral-blood stem-cell transplantation. $\mathrm{N}$ Engl J Med 343, 750, 2000.

4. Jones, E.A., English, A., Henshaw, K., Kinsey, S.E., Markham, A.F., Emery, P. and McGonagle, D. Enumeration and phenotypic characterization of synovial fluid multipotential mesenchymal progenitor cells in inflammatory and degenerative arthritis. Arthritis Rheumatism 50, 817, 2004.

5. Huelsken, J., Vogel, R., Erdmann, B., Cotsarelis, G. and Birchmeier, W. beta-Catenin controls hair follicle morphogenesis and stem cell differentiation in the skin. Cell 105, 533, 2001.

6. Seale, P., Asakura, A. and Rudnicki, M.A. The potential of muscle stem cells. Dev Cell 1, 333, 2001.

7. Gronthos, S., Brahim, J., Li, W., Fisher, L. W., Cherman, N., Boyde, A., DenBesten, P., Robey, P. G. and Shi, S. Stem cell properties of human dental pulp stem cells. J Dent Res 81, 531, 2002.

8. Tondreau, T., Lagneaux, L., Dejeneffe, M., Delforge, A., Massy, M., Mortier, C. and Bron, D. Isolation of BM mesenchymal stem cells by plastic adhesion or negative selection: phenotype, proliferation kinetics and differentiation potential. Cytotherapy 6, 372, 2004.

9. Mueller, S.M. and Glowacki, J. Age-related decline in the osteogenic potential of human bone marrow cells cultured in three-dimensional collagen sponges. J Cell Biochem 82, 583, 2001.

10. Lee, S.Y., Miwa, M., Sakai, Y., Kuroda, R., Matsumoto, T., Iwakura, T., Fujioka, H., Doita, M. and Kurosaka, M. In vitro multipotentiality and characterization of human unfractured traumatic hemarthrosis-derived progenitor cells: A potential cell source for tissue repair. I Cell Physiol 210, 561, 2007.
11. Can, A. and Karahuseyinoglu, S. Concise review: human umbilical cord stroma with regard to the source of fetusderived stem cells. Stem Cells 25, 2886, 2007.

12. Weiss, M.L., Medicetty, S., Bledsoe, A.R., Rachakatla, R.S., Choi, M., Merchav, S., Luo, Y., Rao, M.S., Velagaleti, G. and Troyer, D. Human umbilical cord matrix stem cells: preliminary characterization and effect of transplantation in a rodent model of Parkinson's disease. Stem Cells 24, 781, 2006.

13. Karahuseyinoglu, S., Cinar, O., Kilic, E., Kara, F., Akay, G.G., Demiralp, D.O., Tukun, A., Uckan, D. and Can, A. Biology of stem cells in human umbilical cord stroma: in situ and in vitro surveys. Stem Cells 25, 319, 2007.

14. Lu, L.L., Liu, Y.J., Yang, S.G., Zhao, Q.J., Wang, X., Gong, W., Han, Z.B., Xu, Z.S., Lu, Y.X., Liu, D., Chen, Z.Z. and Han, Z.C. Isolation and characterization of human umbilical cord mesenchymal stem cells with hematopoiesis-supportive function and other potentials. Haematologica 91, 1017, 2006.

15. Wu, K.H., Zhou, B., Lu, S.H., Feng, B., Yang, S.G., Du, W.T., $\mathrm{Gu}$, D.S., Han, Z.C. and Liu, Y.L. In vitro and in vivo differentiation of human umbilical cord derived stem cells into endothelial cells. J Cell Biochem 100, 608, 2007.

16. Campard, D., Lysy, P.A., Najimi, M. and Sokal, E.M. Native umbilical cord matrix stem cells express hepatic markers and differentiate into hepatocyte-like cells. Gastroenterology 134, 833, 2008.

17. Wang, H.S., Hung, S.C., Peng, S.T., Huang, C.C., Wei, H.M., Guo, Y.J., Fu, Y.S., Lai, M.C. and Chen, C.C. Mesenchymal stem cells in the Wharton's jelly of the human umbilical cord. Stem Cells 22, 1330, 2004.

18. Sarugaser, R., Lickorish, D., Baksh, D., Hosseini, M.M. and Davies, J.E. Human umbilical cord perivascular (HUCPV) cells: a source of mesenchymal progenitors. Stem Cells 23, 220, 2005.

19. Baksh, D., Yao, R. and Tuan, R.S. Comparison of proliferative and multilineage differentiation potential of human mesenchymal stem cells derived from umbilical cord and bone marrow. Stem Cells 2007.

20. Mitchell, K.E., Weiss, M.L., Mitchell, B.M., Martin, P., Davis, D., Morales, L., Helwig, B., Beerenstrauch, M., Abou-Easa, K., Hildreth, T., Troyer, D. and Medicetty, S. Matrix cells from Wharton's jelly form neurons and glia. Stem Cells 21, 50, 2003.

21. Kadner, A., Hoerstrup, S.P., Tracy, J., Breymann, C., Maurus, C.F., Melnitchouk, S., Kadner, G., Zund, G. and Turina, M. Human umbilical cord cells: a new cell source for cardiovascular tissue engineering. Ann Thorac Surg 74, S1422, 2002.

22. Kadner, A., Zund, G., Maurus, C., Breymann, C., Yakarisik, S., Kadner, G., Turina, M. and Hoerstrup, S.P. Human umbilical cord cells for cardiovascular tissue engineering: a comparative study. Eur J Cardiothorac Surg 25, 635, 2004.

23. Carlin, R., Davis, D., Weiss, M., Schultz, B. and Troyer, D. Expression of early transcription factors Oct4, Sox2 and Nanog by porcine umbilical cord (PUC) matrix cells. Reprod Biol Endocrinol 4, 8, 2006.

24. Wu, K.H., Zhou, B., Yu, C.T., Cui, B., Lu, S.H., Han, Z.C. and Liu, Y.L. Therapeutic potential of human umbilical cord derived stem cells in a rat myocardial infarction model. Ann Thorac Surg 83, 1491, 2007.

25. Eblenkamp, M., Aigner, J., Hintermair, J., Potthoff, S., Hopfner, U., Jacobs, V., Niemeyer, M. and Wintermantel, E. [Umbilical cord stromal cells (UCSC). Cells featuring osteogenic differentiation potential]. Orthopade 33, 1338, 2004.

26. Bailey, M.M., Wang, L., Bode, C.J., Mitchell, K.E. and Detamore, M.S. A comparison of human umbilical cord matrix 
stem cells and temporomandibular joint condylar chondrocytes for tissue engineering temporomandibular joint condylar cartilage. Tissue Eng 13, 2003, 2007.

27. Wang, L., Seshareddy, K., Weiss, M.L. and Detamore, M.S. Effect of initial seeding density on human umbilical cord mesenchymal stromal cells for fibrocartilage tissue engineering. Tissue Eng Part A Epub ahead of print 1, 2008.

28. Naughton, B.A., San Roman, J., Liu, K., Purchio, A., Pavelec, R. and Rekettye, L. Cells isolated from Wharton's jelly of the human umbilical cord develop a cartilage phenotype when treated with TGF- $\beta$ in vitro. FASEB J 11:A19 (Abstr. No. 108), 1997.

29. Edwards, C.A. and O'Brien, W.D. Modified assay for determination of hydroxyproline in a tissue hydrolyzate. Clin Chim Acta 104, 161, 1980.

30. Livak, K.J. and Schmittgen, T.D. Analysis of relative gene expression data using real-time quantitative PCR and the 2(-Delta Delta C(T)) method. Methods 25, 402, 2001.

31. Troyer, D.L. and Weiss, M.L. Wharton's jelly-derived cells are a primitive stromal cell population. Stem Cells 26, 591, 2008.

32. Detamore, M.S. and Athanasiou, K.A. Evaluation of three growth factors for TMJ disc tissue engineering. Ann Biomed Eng 33, 383, 2005.

33. Nehring, D., Adamietz, P., Meenen, N.M. and Pötner, R. Perfusion cultures and modelling of oxygen uptake with three-dimensional chondrocyte pellets. Biotechnol Techniques 13, 701, 1999.

34. Adesida, A.B., Grady, L.M., Khan, W.S., Millward-Sadler, S.J., Salter, D.M. and Hardingham, T.E. Human meniscus cells express hypoxia inducible factor-1alpha and increased SOX9 in response to low oxygen tension in cell aggregate culture. Arthritis Res Ther 9, R69, 2007.

35. Yoo, J.U., Barthel, T.S., Nishimura, K., Solchaga, L., Caplan, A.I., Goldberg, V.M. and Johnstone, B. The chondrogenic potential of human bone-marrow-derived mesenchymal progenitor cells. J Bone Joint Surg Am 80, 1745, 1998.

Address correspondence to: Michael S. Detamore, Ph.D. Department of Chemical and Petroleum Engineering 4132 Learned Hall, $1530 \mathrm{~W} 15^{\text {th }}$ St University of Kansas Lawrence, KS 66045

E-mail: detamore@ku.edu

Received: July 13, 2008 Accepted: December 3, 2008

Online Publication Date: February 27, 2009 


\section{This article has been cited by:}

1. Kalamegam Gauthaman, Chui-Yee Fong, Arjunan Subramanian, Arijit Biswas, Ariff Bongso. 2010. ROCK Inhibitor Y-27632 Increases Thaw-Survival Rates and Preserves Stemness and Differentiation Potential of Human Wharton's Jelly Stem Cells After Cryopreservation. Stem Cell Reviews and Reports 6:4, 665-676. [CrossRef]

2. Cun-Gang Fan, Qing-jun Zhang, Jing-ru Zhou. 2010. Therapeutic Potentials of Mesenchymal Stem Cells Derived from Human Umbilical Cord. Stem Cell Reviews and Reports . [CrossRef]

3. Chui-Yee Fong, Li-Ling Chak, Arijit Biswas, Jee-Hian Tan, Kalamegam Gauthaman, Woon-Khiong Chan, Ariff Bongso. 2010. Human Wharton's Jelly Stem Cells Have Unique Transcriptome Profiles Compared to Human Embryonic Stem Cells and Other Mesenchymal Stem Cells. Stem Cell Reviews and Reports . [CrossRef]

4. Ashleigh E. Nugent, David A. Reiter, Kenneth W. Fishbein , Denise L. McBurney, Travis Murray , Dorota Bartusik , Sharan Ramaswamy, Richard G. Spencer, Walter E. Horton , Jr. . 2010. Characterization of Ex Vivo-Generated Bovine and Human Cartilage by Immunohistochemical, Biochemical, and Magnetic Resonance Imaging AnalysesCharacterization of Ex Vivo-Generated Bovine and Human Cartilage by Immunohistochemical, Biochemical, and Magnetic Resonance Imaging Analyses. Tissue Engineering Part A 16:7, 2183-2196. [Abstract] [Full Text] [PDF] [PDF Plus]

5. Huiling Cao, Hui Qian, Wenrong Xu, Wei Zhu, Xu Zhang, Yuan Chen, Mei Wang, Yongming Yan, Ying Xie. 2010. Mesenchymal stem cells derived from human umbilical cord ameliorate ischemia/reperfusion-induced acute renal failure in rats. Biotechnology Letters 32:5, 725-732. [CrossRef]

6. F. Hildner, S. Wolbank, H. Redl, M. van Griensven, A. Peterbauer. 2010. How chondrogenic are human umbilical cord matrix cells? A comparison to adipose-derived stem cells. Journal of Tissue Engineering and Regenerative Medicine 4:3, 242-245. [CrossRef] 